\title{
Synergistic effect of Bruton's tyrosine kinase and TNF- $\alpha$ in the regulation of rheumatoid arthritis and underlying mechanisms
}

\author{
JINWAN DU
}

Department of Rheumatology and Immunology, Chongqing Ninth People's Hospital, Beibei, Chongqing 400700, P.R. China

Received April 10, 2021; Accepted September 1, 2021

DOI: $10.3892 / \mathrm{etm} .2021 .11064$

\begin{abstract}
The presence of Bruton's tyrosine kinase (BTK) in macrophages has been recommended as a promising therapeutic target for rheumatoid arthritis (RA). In addition, activated macrophages in the inflamed joints of patients with RA can also produce a plethora of cytokines, such as TNF- $\alpha$. The aim of the present study was to investigate the potential role of BTK and TNF- $\alpha$ in the regulation of RA. The results demonstrated that higher levels of BTK and TNF- $\alpha$ were observed in macrophages in inflamed RA joints compared with those in normal joint tissues. Subsequently, the role of BTK and TNF- $\alpha$ in the regulation of cellular process in inflammatory macrophages was analyzed. It was demonstrated that aberrant expression of BTK and TNF- $\alpha$ in inflammatory macrophages can lead to higher cell proliferation rates. Once the expression of BTK or TNF- $\alpha$ was restricted by using short interfering (si)RNAs ( $\operatorname{siBTK}$ or siTNF- $\alpha$ ), the activity of inflammatory macrophages was significantly downregulated. Of note, when the expression of BTK and TNF- $\alpha$ was simultaneously decreased, the proliferation rate of inflammatory macrophages was inhibited to the greatest extent. Subsequently, the underlying mechanisms through which BTK and TNF- $\alpha$ can regulate RA were investigated. The results demonstrated that BTK mainly regulated the ERK/JNK pathway, while TNF- $\alpha$ mainly affected the inactive rhomboid protein $2 / \mathrm{B}$-cell-activating factor pathway. Finally, animal experiments demonstrated that simultaneous silencing of both BTK and TNF- $\alpha$ can significantly alleviate the symptoms associated with RA.
\end{abstract}

\section{Introduction}

As a systemic inflammatory disorder, rheumatoid arthritis (RA) represents one of the most prevalent autoimmune diseases

Correspondence to: Professor Jinwan Du, Department of Rheumatology and Immunology, Chongqing Ninth People's Hospital, 69 Jialing Village, Beibei, Chongqing 400700, P.R. China E-mail: Dr_Du1976@126.com

Key words: rheumatoid arthritis, Bruton's tyrosine kinase, TNF- $\alpha$, ERK/JNK pathway, inactive rhomboid protein $2 / \mathrm{TNF}-\alpha / \mathrm{B}$-cellactivating factor pathway worldwide (1). The immune system in RA can significantly damage the joints and other tissues, ultimately leading to irreversible joint deformity (1-3). Although advances in understanding the pathogenesis of RA have promoted the development of novel therapeutics against this disease, the detailed cause of RA remains unknown and the prognosis is uncertain (4). A number of previous studies have demonstrated that the development of RA involves the activation of a wide range of immune cells and macrophages $(5,6)$. After their activation, these cells can promote RA development by overexpressing a series of important factors, such as major histocompatibility complex class II, proinflammatory cytokines and growth factors $(7,8)$. Recently, targeted intervention of the inflammatory process by disease-modifying antirheumatic drugs (DMARDs) has been used as a promising strategy for the treatment of RA (9-11). However, the risk of subsequent infections was an unavoidable major concern associated with the long-term use of DMARDs (12).

Bruton's tyrosine kinase (BTK), as one of the members of Tec family of non-receptor tyrosine kinases, has been found to serve a key role in the regulation of $\mathrm{B}$ cells and macrophage kinase $(13,14)$. BTK in macrophages has been recommended as a promising therapeutic target for RA, as it plays a significant role in the polarization of proinflammatory macrophages and the production of proinflammatory cytokines (15-17). A number of previous studies have confirmed that knockdown of BTK expression could effectively ameliorate arthritis by significantly reducing the levels of autoantibodies and cytokines $(18,19)$. However, unavoidable immune suppression was diagnosed during the use of BTK inhibitors, mainly due to the off-target effects (20). Therefore, a novel strategy to specifically deliver BTK inhibitors is urgently needed to effectively treat RA.

In addition to the BTK, activated macrophages in the inflamed joints of patients with RA can also produce a plethora of cytokines, such as TNF- $\alpha$, which serves as the main driver of the vicious cycle of inflammation and tissue damage $(21,22)$. It has been demonstrated that inhibition of TNF- $\alpha$ could significantly alleviate the symptoms of RA and delay its progression $(23,24)$.

However, the detailed role of BTK and TNF- $\alpha$ in the regulation of RA and the underlying mechanisms have yet to be extensively investigated. Therefore, in the present study, the potential effects of BTK and TNF- $\alpha$ in the regulation of RA were examined in macrophages in inflamed RA joints. 
Additionally, the underlying mechanisms through which BTK and TNF- $\alpha$ can regulate RA were also investigated.

\section{Materials and methods}

Materials. The short interfering (si)RNAs for BTK and TNF- $\alpha$ (siBTK with sequence of 5'-AAUCCAGCGCUUUCUCAGCd TdT-3' and siTNF- $\alpha$ with sequence of 5'-AAGAGAACCUGG GAGUAGAUAAGGU-3', respectively) were purchased from Shanghai GenePharma Co., Ltd. The negative control siRNA (siNC, with sequence of 5'-ACGUGACACGUUCGGAGAAd TdT-3') was obtained from the Suzhou Ribo Life Science Co., Ltd. MTT was purchased from MilliporeSigma. DMEM, FBS and penicillin-streptomycin were all from Gibco; Thermo Fisher Scientific, Inc.

Cell culture and transfection. The lipopolysaccharide-induced inflammatory mouse macrophage cell line RAW 264.7 and the normal mouse macrophage cell line Ana-1 were obtained from the American Type Culture Collection. The two cells were cultured in DMEM supplemented with $10 \%$ fetal bovine serum, $100 \mathrm{U} / \mathrm{ml}$ penicillin and $100 \mu \mathrm{g} / \mathrm{ml}$ streptomycin at $37^{\circ} \mathrm{C}$ in a $5 \% \mathrm{CO}_{2} / 95 \%$ air humidified environment incubator (Thermo HERA cell; Thermo Fisher Scientific Inc.).

Transfection of RAW 264.7 cells with various genes were performed using Lipofectamine ${ }^{\circledR} 2000$ (Invitrogen; Thermo Fisher Scientific, Inc.). In brief, the RAW 264.7 cells were seeded into each well of a six-well plate at the density of $1 \times 10^{6}$ cells per well. After that, the cells were respectively incubated with siBTK and siTNF- $\alpha$ at $37^{\circ} \mathrm{C}$ in a $5 \% \mathrm{CO}_{2} / 95 \%$ air humidified environment incubator according to the manufacturer's instructions. After $72 \mathrm{~h}$ of transfection, the aforementioned cells were harvested for further experiments.

Development of collagen-induced arthritis (CIA) mouse model. A total of 50 DBA/ 1 mice aged 6-8 weeks $(20 \pm 2 \mathrm{~g})$ were purchased from Shanghai SIPR-BK Laboratory Animal Co., Ltd. and raised at $25 \pm 1^{\circ} \mathrm{C}$ with humidity of $50-60 \%, 12-\mathrm{h}$ light/dark cycle and free access to food and water. Thereafter, RA mouse models were developed based on the previously reported CIA approach (25). In brief, DBA/1 mice were intradermally injected at the base of the tail with bovine type II collagen (Chondrex, Inc.) at a dose of $5 \mathrm{mg} / \mathrm{kg}$ emulsified by $100 \mu \mathrm{l}$ Complete Freund's Adjuvant (Chondrex, Inc.) on day 0 . After being maintained for 21 days, the mice were again treated with emulsified bovine type II collagen $(5 \mathrm{mg} / \mathrm{kg})$. Then, the CIA scores (26) of the mice were carefully recorded by the following criteria: 0 , normal; 1 , one hind paw or fore paw joint affected or minimal diffuse erythema and swelling; 2 , two hind or fore paw joints affected or mild diffuse erythema and swelling; 3 , three hind or fore paw joints affected or moderate diffuse erythema and swelling; 4 , marked diffuse erythema and swelling or four digit joints affected; 5 , severe diffuse erythema and severe swelling of entire paw, unable to flex digits.

Of note, all the experimental procedures were performed according to the guidelines approved by the Chongqing Ninth People's Hospital (approval no. CQSY201911).

Measurement of cell proliferation. The inhibitory effects of siBTK and siTNF- $\alpha$ on the proliferation of RAW 264.7 cells were determined using an MTT assay. Briefly, stably transfected RAW 264.7 cells were seeded into 96-well plates at a density of $5 \times 10^{3}$ cells per well. After an overnight incubation at $37^{\circ} \mathrm{C}$, the old medium was replaced with fresh medium. After incubation for 24,36 or $48 \mathrm{~h}, 20 \mu \mathrm{l}$ of MTT solution was added into each well followed by further incubation for $4 \mathrm{~h}$. Then, $150 \mu \mathrm{l}$ of dimethyl sulfoxide was added and the absorbance of each well was measured at $490 \mathrm{~nm}$ via a microplate reader (Multiskan MK3; Thermo Fisher Scientific, Inc.).

Measurement of cell colony-forming ability. The effects of siBTK and siTNF- $\alpha$ on the proliferation and clonogenic potential of RAW 264.7 cells were evaluated using a cell colony formation assay. A total of $1 \times 10^{5}$ transfected RAW 264.7 cells were seeded into each well of a 24-well plate and incubated overnight. Then, the old medium in the plates was replaced with fresh medium followed by another $24 \mathrm{~h}$ of incubation. Subsequently, the cells were fixed with $4 \%$ paraformaldehyde for $30 \mathrm{~min}$ at room temperature and cell colony (>50 cells) were quantified by evaluation of absorbance at $570 \mathrm{~nm}$ in a plate reader (Thermo Multiskan MK3; Thermo Fisher Scientific, Inc.) after staining with $1 \%$ crystal violet solution for $30 \mathrm{~min}$ at room temperature. The qualitative analysis was evaluated under a phase-contrast microscope (Leica Microsystems $\mathrm{GmbH})$.

Reverse transcription-quantitative (RT-q)PCR assay. Total RNA from the cells (RAW 264.7 cells or Ana-1 cells) or inflammation tissues was isolated using TRIzol ${ }^{\circledR}$ reagent (Invitrogen; Thermo Fisher Scientific, Inc.) and the miScript Reverse Transcription kit (Qiagen NV) was used to generate the cDNA according to the manufacturer's instructions. Subsequently, the detection of BTK and TNF- $\alpha$ was carried out using the miScript SYBR-Green PCR kit (Qiagen NV) followed by analysis using the ABI 7500 PCR analyzer (Applied Biosystems; Thermo Fisher Scientific, Inc.). Of great importance, the PCR cycles were: Pretreatment for $10 \mathrm{~min}$ at $95^{\circ} \mathrm{C}, 96^{\circ} \mathrm{C}$ for $15 \mathrm{sec}, 64^{\circ} \mathrm{C}$ for $45 \mathrm{sec}(45 \mathrm{cycles}), 96^{\circ} \mathrm{C}$ for $15 \mathrm{sec}, 64^{\circ} \mathrm{C}$ for $1 \mathrm{~min}, 95^{\circ} \mathrm{C}$ for $15 \mathrm{sec}$, a final extension at $75^{\circ} \mathrm{C}$ for $10 \mathrm{~min}$ and held at $4^{\circ} \mathrm{C}$. The relative gene expression of BTK and TNF- $\alpha$ was calculated using the $2^{-\Delta \Delta C q}$ method (27) and normalized with $\beta$-actin. The primer sequence, designed by Invitrogen (Thermo Fisher Scientific, Inc.), used for BTK was F, 5'-TGTTGAAACAGT GGTTCCTGA-3' and R, 5'-TGCTCCATTTCACTGGAC TCT-3'; TNF- $\alpha$ was F, 5'-CAGCCTCTTCTCCTTCCTGA-3' and R, 5'-GGAAGACCCCTCCCAGATAGA- 3 ' and $\beta$-actin was F, 5'-ATGGGCCAGAAAGATGCCTATGT-3' and R, 5'-ATGCCAGGGAACATAGTTGAGCC-3'.

Western blot analysis. The cells (RAW 264.7 cells or Ana-1 cells) were collected and lysed using RIPA lysis buffer (Beyotime Institute of Biotechnology). Subsequently, protein concentration was determined using the BCA detection method. Subsequently, the protein samples (BTK $81.3 \mathrm{kDa}$, TNF- $\alpha 17.4 \mathrm{kDa}$, p-ERK $45 \mathrm{kDa}$, p-JNK $51 \mathrm{kDa}, \mathrm{p}-\mathrm{P} 38$ $43 \mathrm{kDa}$, iRhom $297 \mathrm{kDa}$, BAFF $17 \mathrm{kDa}$ and $\beta$-actin $42 \mathrm{kDa}$ all at $100 \mu \mathrm{g}$ ) were separated by $10 \%$ SDS-PAGE and electrophoretically transferred to PVDF membranes. The membranes were blocked in 5\% skimmed milk for $1 \mathrm{~h}$ at room temperature and were then incubated with primary antibodies against BTK 

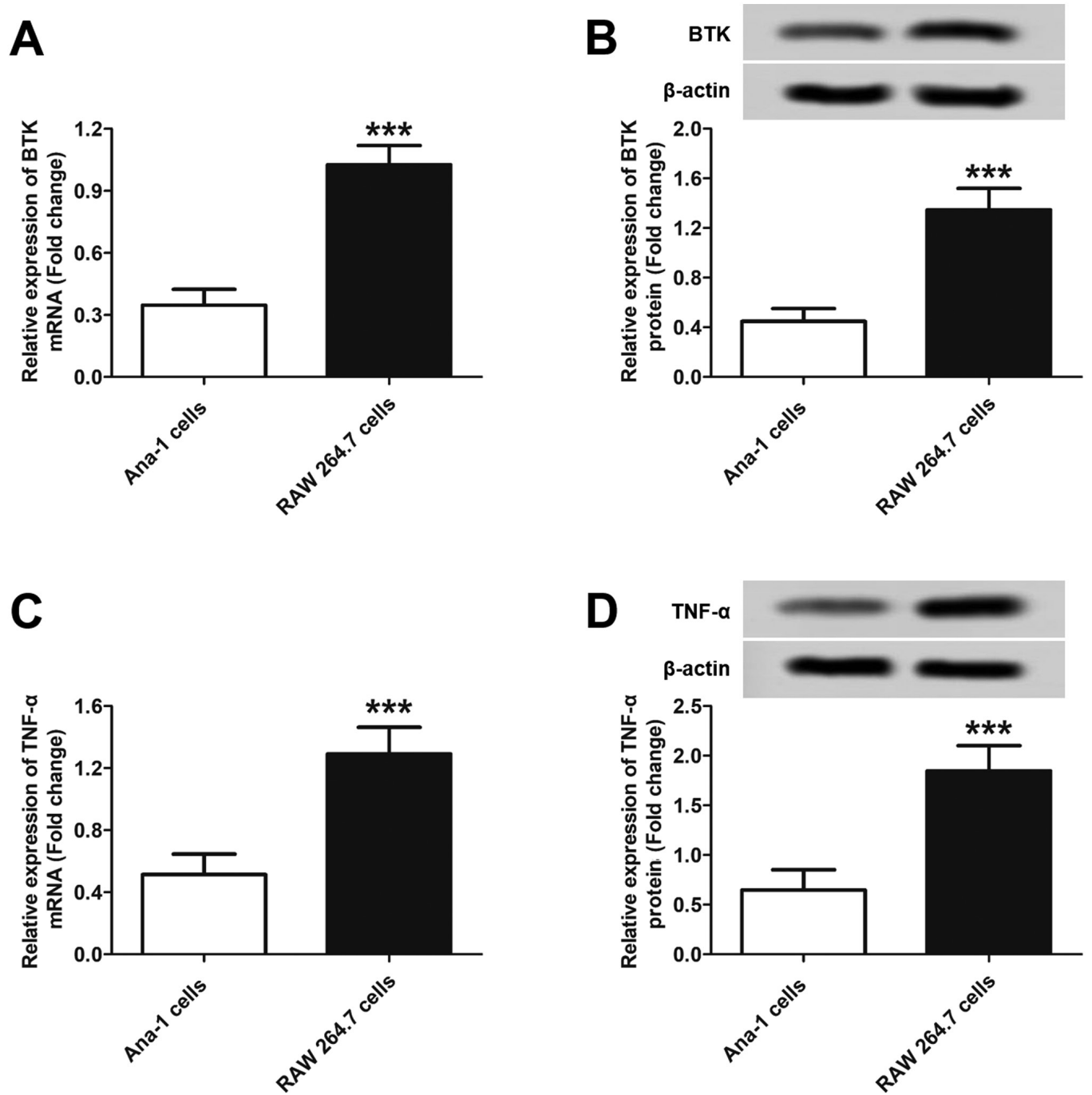

Figure 1. BTK and TNF- $\alpha$ are highly expressed in macrophages from inflamed RA joints. (A) BTK mRNA expression in macrophages from inflamed RA joints and normal macrophages was detected using RT-qPCR experiments. (B) BTK protein expression in macrophages from inflamed RA joints and normal macrophages was detected using western blot assay. (C) TNF- $\alpha$ mRNA expression in macrophages from inflamed RA joints and normal macrophages was detected using RT-qPCR experiments. (D) TNF- $\alpha$ protein expression in macrophages from inflamed RA joints and normal macrophages was detected using western blot assay. ${ }^{* * *} \mathrm{P}<0.001$ vs. Ana-1 cells. BTK, Bruton's tyrosine kinase; RA, rheumatoid arthritis; RT-qPCR, reverse transcription-quantitative PCR.

and TNF- $\alpha$ (cat. nos. ab208937 and ab1793, Abcam; 1:1,500) were added. After an overnight incubation under $4^{\circ} \mathrm{C}$, horseradish peroxidase-conjugated $\mathrm{IgG}$ (cat. no. ab10183, Abcam; $1: 3,000)$ was added at $37^{\circ} \mathrm{C}$. After $1 \mathrm{~h}$, the signals of BTK and TNF- $\alpha$ were visualized using the ECL kit (Merck KGaA) with $\beta$-actin serving as the internal control.

Treatment of CIA mouse models in vivo. The developed CIA mouse models were randomly divided into five groups ( $n=10$ per group) as follows: i) Control, ii) siNC, iii) siBTK, iv) siTNF- $\alpha$ and v) siBTK + siTNF- $\alpha$. The siRNA dose was $2 \mathrm{mg} / \mathrm{kg}$ and mice treated with saline served as the control group. Then, the CIA scores were carefully recorded at the indicated time points $(0,2,4,6,8,10,12$ and 14 days). At the end of the experimental period, all the mice were euthanized by cervical dislocation.

Statistical analysis. Statistical analysis was performed using GraphPad Prism 5 (GraphPad Software, Inc.). $\mathrm{P}<0.05$ was considered to indicate a statistically significant difference. All experiments were performed in triplicates and the data are presented as the mean \pm SD. Unpaired Student's t-test was used for comparisons between two groups and one-way ANOVA with Bonferroni tests was used for multiple-group analysis.

\section{Results}

High expression of BTK and TNF- $\alpha$ is detected in macrophages from inflamed $R A$ joints. To investigate the possible expression of BTK and TNF- $\alpha$ in macrophages from inflamed RA joints, RAW 264.7 inflammatory macrophages were used. Additionally, the levels of BTK and TNF- $\alpha$ were also evaluated in normal Ana-1 macrophages and compared with the results of RAW 264.7 cells. As shown in Fig. 1A, significantly high levels of BTK mRNA were detected in RAW 264.7 cells. However, a relative low expression of BTK mRNA was detected in Ana-1 cells. These results were further confirmed by western blot analysis. As shown in Fig. 1B, the inflammatory macrophages expressed significantly higher BTK protein levels compared with normal macrophages. Semi-quantitative 

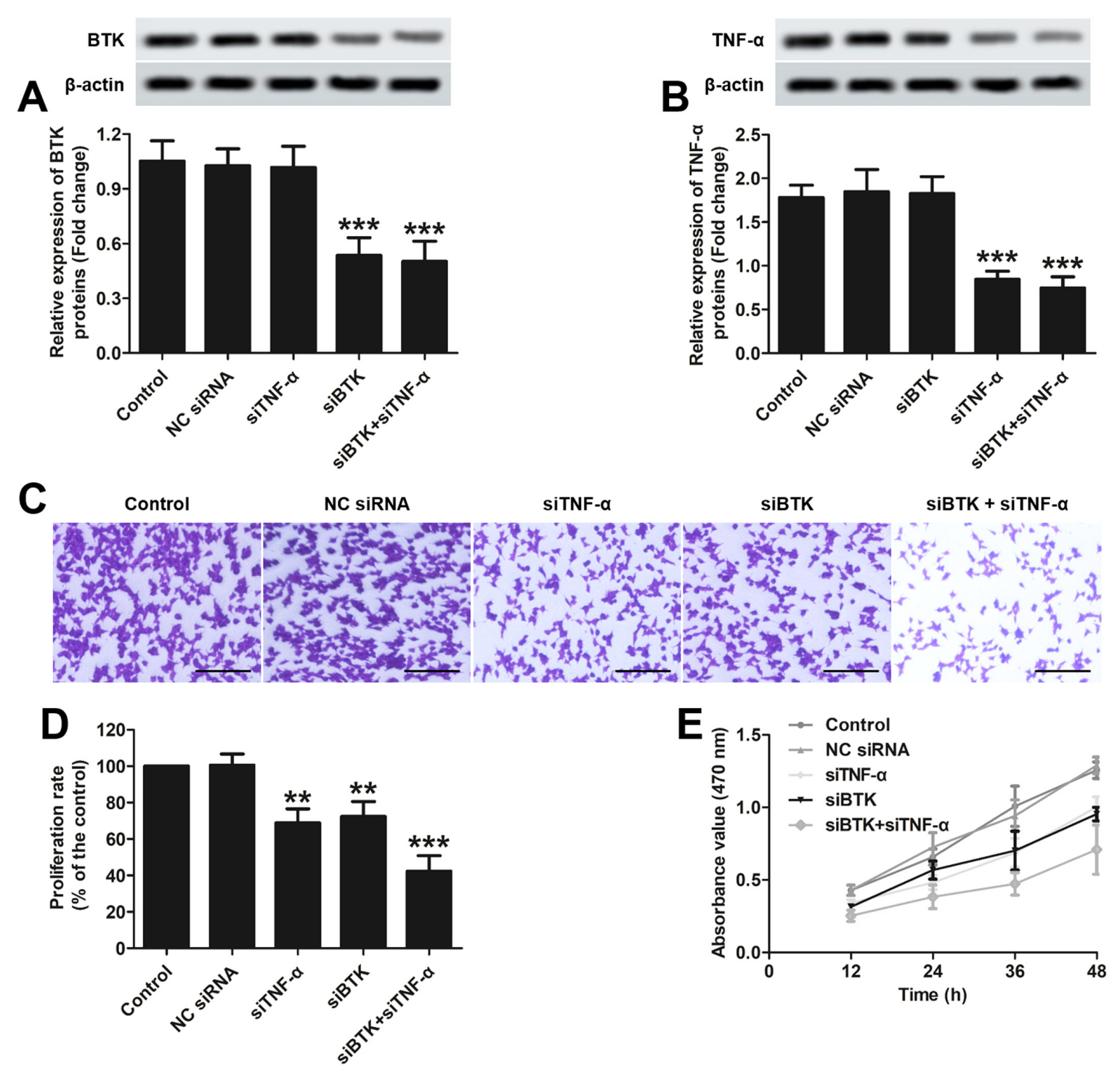

Figure 2. Aberrant expression of BTK and TNF- $\alpha$ promotes the proliferation and clonogenic ability of inflammatory macrophages. (A) BTK and (B) TNF- $\alpha$ protein expression in macrophages from inflamed RA joints and normal macrophages was detected using western blot assays. (C) The colony-forming ability of transfected RAW 264.7 cells was determined using colony formation assays. Scale bar, $100 \mu \mathrm{m}$. (D) Semi-quantitative analysis of the proliferation rate of RAW 264.7 cells. (E) The proliferation of transfected RAW 264.7 cells was determined using an MTT assay. ${ }^{* *} \mathrm{P}<0.01,{ }^{* * * *} \mathrm{P}<0.001 \mathrm{vs}$. control or siNC groups. BTK, Bruton's tyrosine kinase; RA, rheumatoid arthritis; si, short interfering RNA; NC, negative control.

analysis revealed that the BTK protein level in inflammatory macrophages was 3.01-fold that in normal macrophages. As regards TNF- $\alpha$ expression, similar results were obtained, with higher levels of TNF- $\alpha$ mRNA and protein detected in RAW 264.7 cells compared with Ana-1 cells (Fig. 1C and D). Further semi-quantitative analysis revealed that the TNF- $\alpha$ protein level in RAW 264.7 cells was 2.01-fold that in Ana-1 cells. Collectively, the aforementioned results demonstrated that higher levels of BTK and TNF- $\alpha$ may be present in macrophages in inflamed RA joints compared with those in normal joint tissues.

Aberrant expression of BTK and TNF- $\alpha$ promotes the proliferation and clonogenic potential of inflammatory macrophages. The transfection efficacy in RAW 264.7 cells was first evaluated via detection of BTK and TNF- $\alpha$ protein levels using western blotting. As shown in Fig. 2A, cells transfected with siBTK exhibited significantly lower expression of the BTK protein compared with the control group or cells transfected with siNC. However, the cells transfected with siTNF- $\alpha$ exhibited a similar expression level of the BTK protein as the control group or cells transfected with siNC. As regards TNF- $\alpha$ expression, it was demonstrated that the cells transfected with siTNF- $\alpha$ exhibited lower expression of the TNF- $\alpha$ protein compared with the control group or cells transfected with siNC (Fig. 2B). However, the cells transfected with siBTK exhibited slightly lower expression of TNF- $\alpha$ compared with the control group or cells transfected with siNC, thus indicating that silencing of BTK expression exerted a negative regulatory effect on the expression of TNF- $\alpha$.

Subsequently, the inhibitory effects of siBTK and siTNF- $\alpha$ on the colony-forming ability of RAW 264.7 cells was determined using colony formation assay. As shown in Fig. 2C, the cells in the of siBTK and siTNF- $\alpha$ groups displayed a similar extent of crystal violet staining, which was signally lower compared with that in the control and siNC groups. 
A

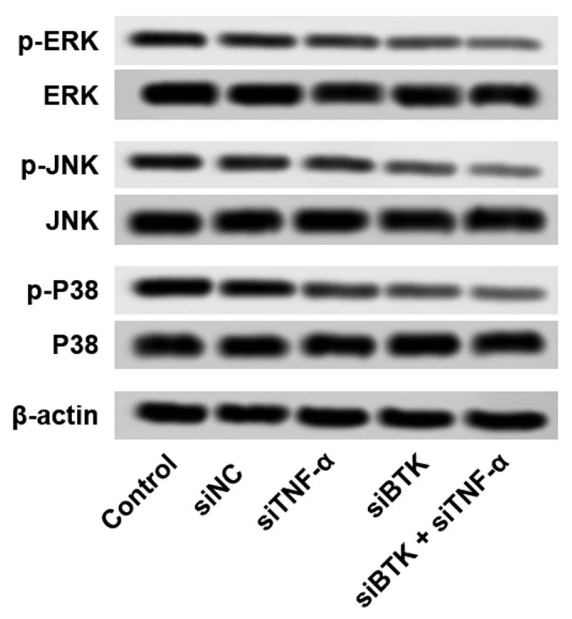

B

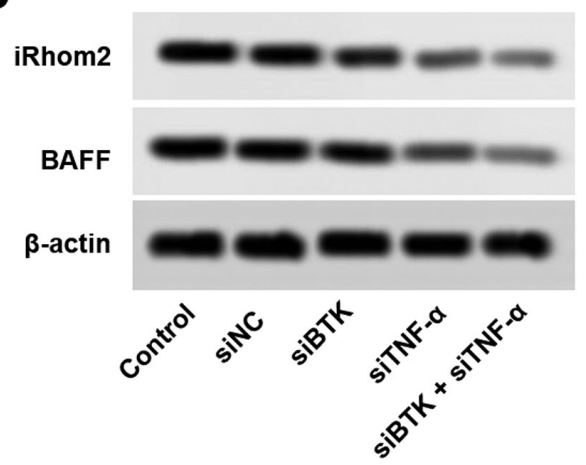

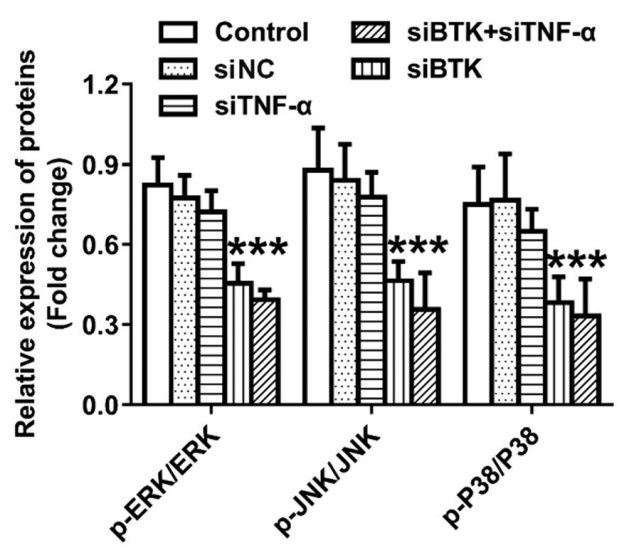

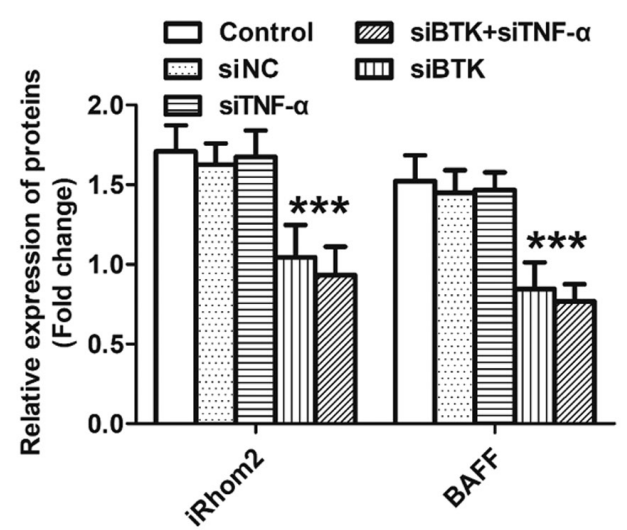

Figure 3. BTK mainly regulates the ERK/JNK pathway and TNF- $\alpha$ mainly regulates the iRhom2/BAFF pathway. (A) ERK, JNK and p38 protein expression in macrophages from inflamed RA joints was detected using western blot assay. (B) iRhom 2 and BAFF protein expression in macrophages from inflamed RA joints and normal macrophages was detected using western blot assay. ${ }^{* * *} \mathrm{P}<0.001$ vs. control or siNC groups. iRhom 2 , inactive rhomboid protein 2 ; BAFF, B-cell-activating factor; BTK, Bruton's tyrosine kinase; RA, rheumatoid arthritis; si, short interfering RNA; NC, negative control.

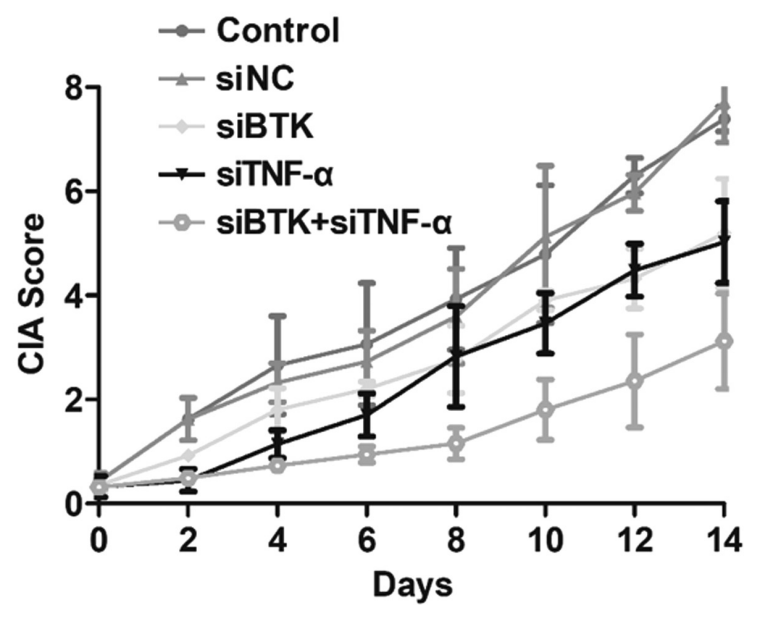

Figure 4. Simultaneously silencing BTK and TNF- $\alpha$ in inflammatory macrophages markedly relieve RA symptoms in vivo. The mouse CIA scores were measured every 2 days post-treatment. BTK, Bruton's tyrosine kinase; RA, rheumatoid arthritis; CIA, collagen-induced arthritis.

Moreover, the cells simultaneously transfected with siBTK and siTNF- $\alpha$ exhibited the lowest extent of crystal violet staining. These results were further confirmed by semi-quantitative analysis (Fig. 2D). Furthermore, the inhibitory effects of siBTK and siTNF- $\alpha$ on the proliferation of RAW 264.7 cells were also determined using MTT assay. As demonstrated in Fig. 2E, similar cell proliferation rates were observed in cells transfected with siBTK and siTNF- $\alpha$, and they were markedly lower compared with those in the control and siNC groups. Furthermore, the proliferation rate of the cells simultaneously transfected with siBTK and siTNF- $\alpha$ was the lowest among all groups. Taken together, these results indicated that BTK and TNF- $\alpha$ play a major role in the proliferation and clonogenic potential of inflammatory macrophages and may exert a synergistic effects.

$B T K$ primarily regulates the ERK/JNK pathway whereas the $T N F-\alpha$ modulates the inactive rhomboid protein 2 (iRhom2)/ $B$-cell-activating factor (BAFF) pathway. Subsequently, the underlying mechanisms through which BTK and TNF- $\alpha$ can promote RA were investigated at the cellular level. As demonstrated in Fig. 3A, silencing of BTK in inflammatory macrophages substantially downregulated the protein expression of ERK, p38 and JNK. However, no significant difference was observed among the control, siNC and siTNF- $\alpha$ groups, thereby suggesting that TNF- $\alpha$ did not affect the ERK/JNK pathway. The mechanisms underlying the action of TNF- $\alpha$ were determined as well. As shown in Fig. 3B, the cells transected with siTNF- $\alpha$, but not siBTK, displayed the lowest protein 
expression of iRhom 2 and BAFF among all the groups. By contrast, there was no significant difference observed among the control, siNC and siBTK groups, thereby indicating that BTK did not exert a regulatory effect on the iRhom2/BAFF pathway. Taken together, these results demonstrated that BTK and TNF- $\alpha$ can induce RA mainly through activation of the ERK/JNK and the iRhom2/BAFF pathways, respectively.

Simultaneously silencing BTK and TNF- $\alpha$ in inflammatory macrophages significantly relieves $R A$ symptoms in vivo. The effects of siBTK and siTNF- $\alpha$ on RA were evaluated in vivo using the developed CIA mouse models. For the experiments, the CIA mice were randomly grouped ( $\mathrm{n}=10$ per group) and treated with saline (control group), siNC, siBTK, siTNF- $\alpha$ or siBTK + siTNF- $\alpha$. Thereafter, the CIA scores of the treated RA mice were carefully observed and recorded every 2 days. As shown in Fig. 4, the CIA scores in the control group rapidly increased. However, after treatment with siBTK or siTNF- $\alpha$, the CIA scores of RA mice were significantly reduced. More importantly, the mice treated with siBTK + siTNF- $\alpha$ displayed the lowest CIA scores among all the groups. These results indicated that simultaneously silencing BTK and TNF- $\alpha$ markedly relieves the RA symptoms in vivo.

\section{Discussion}

A number of previous studies have demonstrated that macrophages, fibroblast-like synoviocytes and dendritic cells, among others, may play pivotal roles in the development of RA by inducing extensive destruction of articular cartilage (28-30). Among these cells, activated macrophages constitute the most prominent cell population in the inflamed RA joints, and markedly affect joint inflammation through regulation of the mRNA expression of various factors (31). Among these, BTK may serve as a potential therapeutic target for RA (19). In addition, certain cytokines produced by the macrophages, such as TNF- $\alpha$, play a critical role in driving inflammation during RA (22). In the present study, it was demonstrated that BTK was highly expressed at the mRNA and protein level in the inflamed RA joints compared with normal joint tissues. Similar results were obtained for the expression of TNF- $\alpha$. These results confirmed the role of BTK and TNF- $\alpha$ in the development of RA.

The MAPK pathway, comprising the ERK, JNK and p38 proteins, is one of the most important signal transduction cascades implicated in the activation of macrophages in RA $(32,33)$. It has been demonstrated that persistent activation of the ERK/JNK pathway may be involved in the development of autoimmune and inflammatory diseases (34). Therefore, the ERK/JNK pathway may serve as an important molecular target for the control of inflammatory diseases. In the present study, it was demonstrated that the activity of the ERK/JNK pathway in the inflammatory macrophages was significantly inhibited by silencing the expression of BTK. Additionally, the downregulation of ERK/JNK pathway-related proteins finally resulted in decreased proliferation of inflammatory macrophages and contributed to the significant improvement of RA symptoms in vivo.

A number of previous studies have demonstrated that TNF- $\alpha$, iRhom 2 and BAFF can play a significant role in the development of RA, and a substantial decrease in TNF- $\alpha$, iRhom 2 and BAFF levels may notably improve the symptoms of
RA $(35,36)$. Of note, the expression of BAFF has been reported to be regulated by TNF- $\alpha$, while the production of TNF- $\alpha$ could be effectively controlled by the activity of iRhom 2 in synovial macrophages (36-38). Based on these results, it was suggested that a positive feedback process exists in the iRhom $2 / \mathrm{TNF}-\alpha / \mathrm{BAFF}$ pathway, and reducing the expression of these genes may be a promising strategy for the treatment of RA. The present study demonstrated that cells transfected with siTNF- $\alpha$, but not siBTK, exhibited the lowest expression of iRhom 2 and BAFF proteins among all groups. By contrast, there were no significant differences observed among cells in the control, NC siRNA and siBTK groups, thereby indicating that BTK did not exert regulatory effects on the iRhom2/BAFF pathway.

In conclusion, the present study demonstrated that BTK and TNF- $\alpha$ are expressed at higher levels in inflamed RA joints compared with normal joint tissues, whereas the aberrant expression of BTK and TNF- $\alpha$ contributed to high proliferation rate and clonogenic potential of inflammatory macrophages. Importantly, the activation of inflammatory macrophages was significantly inhibited by BTK and/or TNF- $\alpha$ silencing. Moreover, the most prominent inhibitory effects on inflammatory macrophages were observed after simultaneous silencing of the expression of both BTK and TNF- $\alpha$. Subsequent mechanistic studies demonstrated that BTK primarily regulated the ERK/JNK pathway, whereas TNF- $\alpha$ modulated the iRhom2/BAFF pathway. In summary, the present study demonstrated that simultaneously silencing both BTK and TNF- $\alpha$ can significantly alleviate the symptoms associated with RA and provide a promising strategy for treatment of RA in the clinical setting. However, due to the limited human and material resources, the complicated relationship between the BTK and TNF- $\alpha$ was not thoroughly investigated in the present study and needs to be studied in future.

\section{Acknowledgements}

Not applicable.

\section{Funding}

No funding was received.

\section{Availability of materials}

The datasets used and/or analyzed during the current study are available from the corresponding author on reasonable request.

\section{Authors' contributions}

JD was the guarantor of integrity of the entire study. JD prepared, edited and reviewed the manuscript. He was also involved in the definition of intellectual content, literature research and responsible for the study design, data acquisition and analysis.

\section{Ethics approval and consent to participate}

The present study was approved by the Research Ethics Committee of Chongqing Ninth People's Hospital (approval no.CQSY201911). 


\section{Patient consent for publication}

Not applicable.

\section{Competing interests}

The author declares that they have no competing interests.

\section{References}

1. De Cock D and Hyrich K: Malignancy and rheumatoid arthritis: Epidemiology, risk factors and management. Best Pract Res Clin Rheumatol 32: 869-886, 2018.

2. Wasserman AM: Diagnosis and management of rheumatoid arthritis. Am Fam Physician 84: 1245-1252, 2011.

3. Wasserman A: Rheumatoid arthritis: Common questions about diagnosis and management. Am Fam Physician 97: 455-462, 2018.

4. McInnes IB and Schett G: The pathogenesis of rheumatoid arthritis. N Engl J Med 365: 2205-2219, 2011.

5. Croia C, Bursi R, Sutera D, Petrelli F, Alunno A and Puxeddu I: One year in review 2019: Pathogenesis of rheumatoid arthritis. Clin Exp Rheumatol 37: 347-357, 2019.

6. Udalova IA, Mantovani A and Feldmann M: Macrophage heterogeneity in the context of rheumatoid arthritis. Nat Rev Rheumatol 12: 472-485, 2016.

7. Rana AK, Li Y, Dang Q and Yang F: Monocytes in rheumatoid arthritis: Circulating precursors of macrophages and osteoclasts and, their heterogeneity and plasticity role in RA pathogenesis. Int Immunopharmacol 65: 348-359, 2018

8. Huang QQ, Birkett R, Doyle R, Shi B, Roberts EL, Mao Q and Pope RM: The role of macrophages in the response to TNF inhibition in experimental arthritis. J Immunol 200: 130-138, 2018

9. de La Forest Divonne M, Gottenberg JE and Salliot C: Safety of biologic DMARDs in RA patients in real life: A systematic literature review and meta-analyses of biologic registers. Joint Bone Spine 84: 133-140, 2017.

10. Massalska M, Maslinski W and Ciechomska M: Small molecule inhibitors in the treatment of rheumatoid arthritis and beyond: Latest updates and potential strategy for fighting COVID-19. Cells 9: 1876, 2020.

11. Ternant D, Bejan-Angoulvant T, Passot C, Mulleman D and Paintaud G: Clinical pharmacokinetics and pharmacodynamics of monoclonal antibodies approved to treat rheumatoid arthritis. Clin Pharmacokinet 54: 1107-1123, 2015.

12. Accortt NA, Bonafede MM, Collier DH, Iles J and Curtis JR: Risk of subsequent infection among patients receiving tumor necrosis factor inhibitors and other disease-modifying antirheumatic drugs. Arthritis Rheumatol 68: 67-76, 2016.

13. Lv J, Wu J, He F, Qu Y, Zhang Q and Yu C: Development of Bruton's tyrosine kinase inhibitors for rheumatoid arthritis. Curr Med Chem 25: 5847-5859, 2018.

14. Hendriks RW, Yuvaraj S and Kil LP: Targeting Bruton's tyrosine kinase in B cell malignancies. Nat Rev Cancer 14: 219-232, 2014

15. Norman P: Investigational Bruton's tyrosine kinase inhibitors for the treatment of rheumatoid arthritis. Expert Opin Investig Drugs 25: 891-899, 2016.

16. Hsu J, Gu Y, Tan SL, Narula S, DeMartino JA and Liao C: Bruton's Tyrosine Kinase mediates platelet receptor-induced generation of microparticles: A potential mechanism for amplification of inflammatory responses in rheumatoid arthritis synovial joints. Immunol Lett 150: 97-104, 2013.

17. Whang JA and Chang BY: Bruton's tyrosine kinase inhibitors for the treatment of rheumatoid arthritis. Drug Discov Today 19: 1200-1204, 2014.

18. Di Paolo JA, Huang T, Balazs M, Barbosa J, Barck KH, Bravo BJ, Carano RA, Darrow J, Davies DR, DeForge LE, et al: Specific Btk inhibition suppresses B cell- and myeloid cell-mediated arthritis. Nat Chem Biol 7: 41-50, 2011.

19. Wu J, Zhu Z, Yu Q and Ding C: Tyrosine kinase inhibitors for the treatment of rheumatoid arthritis: Phase I to II clinical trials. Expert Opin Investig Drugs 28: 1113-1123, 2019.
20. Erickson RI, Schutt LK, Tarrant JM, McDowell M, Liu L, Johnson AR, Lewin-Koh SC, Hedehus M, Ross J, Carano RA, et al: Bruton's tyrosine kinase small molecule inhibitors induce a distinct pancreatic toxicity in rats. J Pharmacol Exp Ther 360: 226-238, 2017.

21. Gowhari Shabgah A, Shariati-Sarabi Z, Tavakkol-Afshari J, Ghasemi A, Ghoryani M and Mohammadi M: A significant decrease of BAFF, APRIL, and BAFF receptors following mesenchymal stem cell transplantation in patients with refractory rheumatoid arthritis. Gene 732: 144336, 2020.

22. Li J, Hsu HC and Mountz JD: Managing macrophages in rheumatoid arthritis by reform or removal. Curr Rheumatol Rep 14: 445-454, 2012.

23. Moura RA, Quaresma C, Vieira AR, Gonçalves MJ, PolidoPereira J, Romão VC, Martins N, Canhão H and Fonseca JE: B-cell phenotype and IgD-CD27- memory B cells are affected by TNF-inhibitors and tocilizumab treatment in rheumatoid arthritis. PLoS One 12: e0182927, 2017.

24. Schiff M, Combe B, Dörner T, Kremer JM, Huizinga TW, Veenhuizen M, Gill A, Komocsar W, Berclaz PY, Ortmann R, et al: Efficacy and safety of tabalumab, an anti-BAFF monoclonal antibody, in patients with moderate-to-severe rheumatoid arthritis and inadequate response to TNF inhibitors: Results of a randomised, double-blind, placebo-controlled, phase 3 study. RMD Open 1: e000037, 2015.

25. Zhao G, Liu A, Zhang Y, Zuo ZQ, Cao ZT, Zhang HB, Xu CF and Wang J: Nanoparticle-delivered siRNA targeting Bruton's tyrosine kinase for rheumatoid arthritis therapy. Biomater Sci 7: 4698-4707, 2019

26. Chang BY, Huang MM, Francesco M, Chen J, Sokolove J, Magadala P, Robinson WH and Buggy JJ: The Bruton tyrosine kinase inhibitor PCI-32765 ameliorates autoimmune arthritis by inhibition of multiple effector cells. Arthritis Res Ther 13: R115, 2011.

27. Cao Y, Lu G, Chen X, Chen X, Guo N and Li W: BAFF is involved in the pathogenesis of IgA nephropathy by activating the TRAF6/NF- $\mathrm{B}$ signaling pathway in glomerular mesangial cells. Mol Med Rep 21: 795-805, 2020.

28. O'Neil LJ and Kaplan MJ: Neutrophils in rheumatoid arthritis: breaking immune tolerance and fueling disease. Trends Mol Med 25: 215-227, 2019.

29. Calabresi E, Petrelli F, Bonifacio AF, Puxeddu I and Alunno A: One year in review 2018: Pathogenesis of rheumatoid arthritis. Clin Exp Rheumatol 36: 175-184, 2018.

30. Boissier MC, Semerano L, Challal S, Saidenberg-Kermanac'h N and Falgarone G: Rheumatoid arthritis: From autoimmunity to synovitis and joint destruction. J Autoimmun 39: 222-228, 2012.

31. Kurowska-Stolarska M and Alivernini S: Synovial tissue macrophages: Friend or foe? RMD Open 3: e000527, 2017.

32. Thalhamer T, McGrath MA and Harnett MM: MAPKs and their relevance to arthritis and inflammation. Rheumatology (Oxford) 47: 409-414, 2008

33. Schett G, Tohidast-Akrad M, Smolen JS, Schmid BJ, Steiner CW, Bitzan P, Zenz P, Redlich K, Xu Q and Steiner G: Activation, differential localization, and regulation of the stress-activated protein kinases, extracellular signal-regulated kinase, c-JUN $\mathrm{N}$-terminal kinase, and p38 mitogen-activated protein kinase, in synovial tissue and cells in rheumatoid arthritis. Arthritis Rheum 43: 2501-2512, 2000.

34. Salojin KV, Owusu IB, Millerchip KA, Potter M, Platt KA and Oravecz T: Essential role of MAPK phosphatase-1 in the negative control of innate immune responses. J Immunol 176: 1899-1907, 2006.

35. Shabgah AG, Shariati-Sarabi Z, Tavakkol-Afshari J and Mohammadi M: The role of BAFF and APRIL in rheumatoid arthritis. J Cell Physiol 234: 17050-17063, 2019.

36. Wei F, Chang Y and Wei W: The role of BAFF in the progression of rheumatoid arthritis. Cytokine 76: 537-544, 2015.

37. Lichtenthaler SF: iRHOM2 takes control of rheumatoid arthritis. J Clin Invest 123: 560-562, 2013

38. Siggs OM, Xiao N, Wang Y, Shi H, Tomisato W, Li X, Xia Y and Beutler B: iRhom2 is required for the secretion of mouse TNFa. Blood 119: 5769-5771, 2012. 